\title{
Maximum likelihood method based on specimen information reconstruction and life equivalent principle for P-S-N curve fitting
}

\author{
Wenfei Liu ${ }^{1}$, Yongju Zhang ${ }^{1}$, Liwen $\mathrm{He}^{1}$, Zhixiong $\mathrm{Gao}^{1}$, and Xiongtao $\mathrm{Pu}^{1}$ \\ ${ }^{1}$ Affiliation not available
}

April 28, 2020

\begin{abstract}
In order to improve the accuracy of the relationship between standard deviation and stress with a small number of fatigue test data, the specimen information reconstruction method is proposed in this paper. Meanwhile, the new maximum likelihood method is designed for P-S-N curve fitting, which is based on the specimen information reconstruction and the life equivalent principle. According to the main type of welded joint on the 80t gondola car body, the T-joint specimens were fabricated and tested, then the P-S-N curve is fitted and extrapolated. Finally, according to the result of measured stress spectrum on $80 \mathrm{t}$ gondola car body, it is verified that the accuracy of the P-S-N curve fitted in this paper is higher, and it is indicate that maximum likelihood method based on specimen information reconstruction and life equivalent principle is a better way for P-S-N curve fitting, especially for the small number of fatigue test data.
\end{abstract}

\section{Hosted file}

Maximum likelihood method based on specimen information reconstruction.docx available at https: //authorea.com/users/311925/articles/442565-maximum-likelihood-method-based-on-specimen-informationreconstruction-and-life-equivalent-principle-for-p-s-n-curve-fitting 